\title{
Fibrin deposition in pregnancy
}

\author{
A. L. STALKER \\ From the Department of Pathology, University of Aberdeen
}

There is now considerable evidence, both direct and indirect, that intravascular coagulation occurs in eclampsia and preeclampsia. There is still debate, however, about its timing, significance and mechanism of production and also about the possibility that it occurs to a minor degree in normal pregnancy. It is the purpose of this paper to focus attention on these lesser grades of intravascular coagulation.

The whole concept of intravascular coagulation as an 'intermediary mechanism of disease' (McKay, 1965) can be a difficult one for histopathologists. Classic severe examples, such as may follow placental abruption or fulminating septicaemia, are generally obvious on study of tissue sections but the problems begin when the process operates at an altogether lower tempo and to a lesser degree.

A study of intravascular fibrin deposition in pregnancy is rewarding not only for its own sake but also because it is an example of the wider issues that arise when intravascular coagulation is considered as a component of pathogenesis in a whole range of conditions.

It is first necessary to evaluate the so-called dynamic balance between coagulation and fibrinolysis and then the validity of the methods by which we detect fibrin deposition. Thereafter factors that possibly play a part in intravascular coagulation in pregnancy can be better assessed and provisional conclusions reached on its significance.

\section{The Dynamic Equilibrium between Clotting and Lysis}

Although fibrinolysis has been recognized for almost a century it is only relatively recently that coagulation and fibrinolysis have been regarded as being in dynamic equilibrium to maintain the integrity and patency of the vascular system in the normal life of the individual. Certainly the occurrence of plasma plasminogen activator and serum fibrin degradation products (FDP) in normal subjects is in keeping with such a hypothesis. So, too, is the reduction of fibrinolytic activity generally found in patients with thrombotic conditions. Fibrinolytic inhibitors used in therapy may cause widespread intravascular thrombosis. Impaired coagulation is not, on the other hand, necessarily associated with a corresponding reduction in fibrinolysis.

From the rheological standpoint Copley (1957, 1960) has claimed that a layer of material such as fibrin is a necessary lubricant lining to facilitate vascular perfusion of capillaries. Such a layer has never been demonstrated but the protagonists of this endoendothelial fibrin layer theory claim, of course, that this is due to its continuing lysis and reformation. The work of Todd $(1959,1971)$ demonstrates that fibrinolytic potential is a normal characteristic of endothelium depending upon its site in the vasculature.

Fibrin degradation products act as a stimulus to fibrinogen release and subsequent synthesis by the liver (Barnhart et al, 1970; Simpson et al, 1973). This further suggests that a balance exists but strict proof is still lacking. There can, however, be little doubt that there is an interaction between the coagulation and fibrinolytic systems which can be viewed loosely as an equilibrium. The linkage between inflammation, immunity and haemostasis through the kinin, complement, fibrinolytic and coagulation systems (Ogston, 1973) is a rapidly developing growth point of considerable physiological complexity and pathological importance.

\section{The Histological Demonstration of Coagulation and Fibrinolysis}

The main point the practising histopathologist will often wish to establish from his biopsy or necropsy material in suspected cases of intravascular coagulation is whether or not coagulation has indeed been triggered. This may not always be so simple as it sounds.

Changes in blood platelets, such as adhesion, aggregation and alteration of platelet morphology, would provide the most convincing evidence of the inception of coagulation. However, platelet studies are histologically difficult by optical microscopy. Relatively trivial procedures induce striking changes in platelet ultrastructure, which is the proper level for definitive studies on the platelet. It is thus highly 
unlikely that postmortem material will be suitable. The possibility that biopsy studies, especially renal, may be more rewarding must be kept in mind.

For all practical purposes we are concerned, therefore, with the identification of fibrin. Our knowledge of the fine structure of fibrinogen and fibrin is still limited (Doolittle, 1973) but opinion favours the trinodal model of fibrinogen (Hall and Slayter, 1959). In this, after fibrinopeptide cleavage, there is believed to be end-to-end union of molecules to form fibrin monomer, subsequent side-to-side polymerization of monomers to form fibrils and eventual stabilization of these by factor XIII.

In a continuing project in this department (Huxley, Scott, and Stalker, unpublished) the formation and degradation of fibrin is under investigation in controlled conditions by optical and electron microscopy.

On ultrastructural study of fibrinogen by negative staining techniques the validity of the trinodal model cannot yet be confirmed; it does, however, seem to accord with the observations more closely than any of the other proposed models. When fibrin forms in vitro the characteristic cross-striping of $24 \mathrm{~nm}$ periodicity is seen but it is worth noting that a $\pm 4.5^{\circ}$ tilt of the section can obliterate this. It is thus not surprising that this cross striation can be seen in only a small proportion (about $10 \%$ ) of fibrin fibres on random sampling.

The effect of motion during the clotting process is often overlooked. In fibrin clots forming during agitation we rarely find striation of the much more closely packed fibres.

Activation of the fibrinolytic system normally accompanies coagulation if plasminogen activator is present. Plasminogen and fibrinogen coexist in plasma. It is important therefore to consider the effects of concomitant lysis on the structure of fibrin as it forms. This is, of course, the presumed natural event. Striking changes in fibrin morphology occur rapidly when it is acted upon by plasmin. Breaks occur in the fibres which also show irregular fraying and loss of cross striation. Similar changes can be seen if FDP are present during fibrin formation (Huxley et al, 1975). Much is known of the detail of fibrin degradation through a range of breakdown products (Marder, 1971). We have studied these FDP ultrastructurally but have, not unexpectedly, failed to characterize even the early large fragment $X$ let alone the late small fragments $D$ and $E$.

For all of the reasons given it is not surprising, therefore, that the so-called typical cross striation of fibrin is rarely seen on electron microscopy in tissue sections even when there is good reason on other grounds to suppose that fibrin is present.

Turning to special staining for fibrin on optical microscopy, the literature is totally confusing and few accounts take heed of even the little that is known of fibrin formation and lysis at the molecular level. We have tested 10 well known fibrin staining methods in the early stages of fibrin formation (from $10 \mathrm{~min}$ to $48 \mathrm{hr}$ ) and have not reached firm conclusions. We agree with Gitlin and Craig (1957) that other substances incorporated in fibrin clots, notably albumin, play an enhancing part in the staining reactions. This may explain why fibrin in inflammatory exudates stains more readily. In clots formed from purified fibrinogen by thrombin no staining method has given a consistently positive result. In clots formed from plasma the pattern of staining is notably capricious; its occurrence is irregular as is its depth; the effect of agitation during clot formation varies from stain to stain; there is similar variation if coexisting lysis is present. We have not been able to explain these findings. In many, but not all, aspects of fibrin staining we agree with the comprehensive study of Moe and Abildgaard (1969). We not do however favour the PTAH technique. If we are driven to declare a preference in this imperfect situation we would opt for the MSB method (Lendrum et al, 1962) although we have reservations about its non-specific staining of high molecular weight globulins, lipoproteins and precipitated fibrinogen.

Demonstration by immunofluorescence is probably the most specific and sensitive of the methods for staining fibrinogen, fibrin, and, to a certain extent, their breakdown products. However, even here there is still lack of knowledge about the precise nature of what is being stained, such is the immunological overlap. It is thus not possible to say whether a positive reaction is being given by fibrinogen that is abnormally adherent to a vessel wall or has seeped into it, by fibrin-be it monomer or polymer (stabilized or not) - or even by fibrin that has lysed up to an undetermined degradation point.

It should therefore be evident at this stage that our methods for recognition of fibrin histologically lack the sure foundation of knowledge of its molecular configuration. When this is fully known rapid advances in our techniques will follow.

The extent to which fibrinolysis has occurred in a tissue or vasculature cannot be determined histologically but the capacity of a tissue or vascular wall to produce plasminogen activator can, of course, be demonstrated by the well known Todd (1959) fibrin autograph technique. This works well in our experience, both in biopsy and necropsy material. The main difficulty lies in quantification. Disappointingly little has been done using this method. Studies that have been made, for example in the skin (Ryan, 1972), have shown its value in assessing 
reduction in plasminogen activator produced by endothelial cells.

Finally in this section mention must be made of the classic paper by Mole (1948) on postmortem fibrinolysis. Pathologists are well aware of this phenomenon but do not sufficiently take it into account. Precise measurement of the speed and extent of postmortem lysis cannot be made until we have surer methods for identifying fibrin and its products but they are considerable. What we cannot, however, ignore is the implication for demonstration of fibrin in postmortem material. This is discussed in the next section.

\section{Intravascular Coagulation in the Light of the Technical Limitations Described}

It is evident that caution is required in pronouncing upon the presence or absence of intravascular fibrin in histological sections. In the great majority of instances, such as occlusive or mural thrombi, the amount of fibrin is considerable, and it can be readily demonstrated by one or other of the methods described. But in lesser examples a negative or equivocal reaction given by eosinophilic intravascular material, fibrillar or amorphous, does not mean an indubitable negative. The limitations of our techniques and the possibility of postmortem fibrinolysis cannot be disregarded. Similarly the occurrence of a few microthrombi of demonstrable fibrin does not necessarily mean that this was the total extent of the intravascular formation of fibrin; it could have been notably greater.

These possibilities are of much consequence in the histological diagnosis of intravascular coagulation. In severe examples of this, such as in placental abruption or amniotic fluid embolism, we generally find abundant intravascular fibrin although occasionally the amount is less than might have been expected. It is, however, in the lesser degrees of intravascular coagulation that it can be extremely difficult to prove that fibrin deposition has occurred. Problem cases in the non-obstetric field can include Gram-negative septicaemia, fat and air embolism, burns and acute pancreatitis. If the possibility of intravascular coagulation has been considered clinically and evidence of elevated serum FDP levels, reduced platelet count, or distorted red cells in the peripheral blood, is available greater importance can be attached to quite scanty histological findings and a truer idea of the magnitude of the intravascular coagulation process can be obtained. Unfortunately such supporting evidence is all too often missing. In such circumstances the only other indicator that might prove of value is the appearance in the vascular tree of darkly staining haematoxylinophil bodies.
These have been shown (Brown et al, 1969) to be extruded nuclei of endothelial cells; they tend to aggregate in the lung where they form irregular nuclear masses; if associated with granular material in the microvasculature they may provide a useful pointer to an episode of recent intravascular coagulation (Simpson et al, 1971; Donald, 1972; Goodall, 1973). There is current interest in the possibility that swelling and blebbing of endothelial cell cytoplasm, culminating in nuclear loss, might represent a response by the endothelial cell that is releasing plasminogen activator in relation to adjacent deposition of fibrin. Such swelling and blebbing was described by Brown et al (1969) in their defibrination studies. It seems perhaps more likely that the change is a non-specific reaction to a wide range of injurious stimuli. Nevertheless under certain special conditions the presence of the shed nuclei may be diagnostically helpful.

In summary, the minor forms of intravascular coagulation may be difficult to detect histologically and supporting haematological evidence is often needed. Even this may be lacking or imperfect if one considers the possibility that the coagulation process is episodic. This must affect our thinking on many of the conditions in which it is thought that intravascular coagulation plays some part. Failure to demonstrate fibrin deposition may be taken, quite wrongly, to rule out this process altogether but the uncritical assumption that intravascular coagulation has occurred must also be avoided since this can lead to glib and oversimplistic explanations of pathogenesis. Technical advances will surely help us with this problem in time. Meantime we do well to look at natural disease processes for examples of a hypothetical broad spectrum of intravascular coagulation. One such is, I believe, to be found in eclampsia, preeclampsia and normal pregnancy.

\section{Fibrin Deposition in Pregnancy}

Extensive and widespread deposition of fibrin in full-blown eclampsia has been described fully by Govan $(1954,1973)$ and reemphasized by him in this symposium. The resultant microvascular obstruction can cause parenchymal necrosis.

In preeclampsia percutaneous renal biopsy-or for that matter any other biopsy-is not often performed in Britain so that opportunities for histological investigation are few. There have, however, been conclusive reports from USA (Pirani et al, 1963; Vassalli et al, 1963) that leave no doubt that fibrin deposition occurs in the renal glomerular capillaries in preeclampsia. The glomerular subendothelial deposits of fibrin, with endothelial cell swelling, have also been shown by Thomson et al (1972) to be 
similar in preeclampsia and in placental abruption with proven intravascular coagulation. Fibrin formed in vitro from the plasma of preeclamptic women is ultrastructurally of the frayed and irregular pattern found when FDP are present in excess (Huxley et al, 1975).

In both eclampsia and preeclampsia changes have been found in the coagulation and fibrinolytic systems. They have been reviewed by Bonnar (1973) and restated in this symposium. They point to the occurrence of intravascular coagulation with elevated serum FDP levels, thrombocytopenia and depressed fibrinolytic activity; these vary in extent which suggests an episodic phenomenon of altering tempo and degree.

The histological and haematological findings together thus indicate conclusively that intravascular coagulation is part of the mechanism of this disease process.

Turning to the possible other end of the spectrum -normal pregnancy-there is no histological evidence that fibrin deposition, other than in the special case of the placenta, has been observed, except perhaps in one instance. Vassalli et al (1963) showed by immunofluorescent studies that fibrin was consistently found in the glomerular capillaries of preeclamptic patients. They add: 'The impression was gained that the glomeruli of patients with normal pregnancy showed an equivocal increase in fibrin as compared with normal non-pregnant patients'. There is abundant haematological evidence, however, that the coagulation/fibrinolysis equilibrium is altered in favour of procoagulant activity. There is increase in clotting factors, especially fibrinogen, and depression of activation of fibrinolysis (Bonnar, 1973). The slight but progressive rise in serum FDP through the trimesters of pregnancy (Woodfield et al, 1968) points to the likely occurrence of low grade intravascular coagulation in normal pregnancy. Further, it is also well established that pregnancy potentiates the experimental Shwartzman reaction; this enhancement of fibrin deposition is achieved by depression of the fibrinolytic system, either by drugs or by prior overload of fibrin to be cleared; the latter is the presumed mechanism in pregnancy.

It seems reasonable, therefore, to take the view that the prominent changes of the eclampsia/ preeclampsia picture scale down into minor changes in normal pregnancy. Histological studies of biopsy material obtained in pregnancy should be made when opportunity affords; they will be of greater value when our techniques for demonstrating fibrin improve. This strict histological proof of the occurrence of minor intravascular coagulation in normal pregnancy is still awaited.

\section{Possible Aetiology of Intravascular Coagulation in Pregnancy}

Because coagulation and fibrinolysis factors return to normal after parturition and the features of preeclampsia subside once delivery is accomplished it is in the feto-placental unit that any explanation for intravascular coagulation in pregnancy most probably lies. Several possibilities have been suggested; there may be others.

Entry of large amounts of placental thromboplastin into the maternal circulation after placental trauma was fully described by Schneider $(1952,1959)$. Episodic entry of much smaller, but variable, amounts is essentially the same process. It has been demonstrated experimentally after minor placental injury by Brown and Stalker (1969) and related to the Young and Griffith hydrodynamic theory of parenchymatous tissue embolism (Stalker, 1973). Activation of coagulation, due presumably to thromboplastin release, at the side of placental separation in the human has been proved by the work of Bonnar et al (1970) on uterine vein blood. Whether slight damage occurs normally during the development of the placenta is as yet unproven. It would be suprising if it did not happen. Certainly intervillous thrombosis, marking breaks in villi, is present in a large proportion of placentae in normal pregnancy and can be related to fetal red cell detection in the maternal circulation (Banti Devi et al, 1968). Greater degrees of injury, such as placental infarction, are common in preeclampsia but it could be argued that this is a later consequence of intravascular coagulation, and perhaps part of a vicious circle, rather than a truly primary event.

Another possibility is invasion of the maternal blood vessels by cytotrophoblast as part of the progressive physiological adaptation of the uterine spiral arteries to permit a large increase in blood flow to the placental intervillous space (Brosens et al, 1967). It would seem, however, that local inhibition of fibrinolysis is a more prominent effect of this in view of the fibrin deposition that occurs in the walls of these much enlarged channels.

An oversimplification may be the possibility that the maternal intervillous spaces are virtually the equivalent of a giant haemangioma. It is known that low-grade intravascular coagulation occurs in patients with such haemangiomata as a result either of stasis and thrombosis or of red cell injury in a turbulent area of the circulation. Could this be all that is necessary to trigger coagulation in normal pregnancy?

Hormonal possibilities must also be considered. The feto-placental unit produces oestrogen and progestogen in abundance and the trophoblast has 
been shown to produce a range of pregnancyspecific proteins. All of these may greatly modify the coagulation/fibrinolysis equilibrium. The exact mode of action of steroids on this is not known. They tend to enhance coagulant activity whereas the pregnancy specific proteins may be more related to depression of fibrinolysis, but this is speculative.

As another possibility, intravascular coagulation may be related to an immunological mechanism. It has been suggested by Robertson et al (1967) that the uterine vascular lesions of preeclampsia (acute 'atherosis') are similar to those found in transplant rejection. Triggering of coagulation by circulating immune complexes is well known and Petrucco et al (1974) have shown that complement and immunoglobulins, as well as fibrin, are deposited in renal glomerular capillaries in preeclampsia. But fuller proof of an immunological basis is still required.

That spasm of uterine arteries is primarily responsible for fetal placental damage and subsequent intravascular coagulation seems improbable in view of the physiological gross alteration in the spiral arteries of the placental bed described above (Brosens et al, 1967). Such vessels, devoid of their musculo-elastic wall, could scarcely respond to vasomotor stimuli. However, proximal vessels could react to such stimuli and spasm of these may have a part to play. Further work on the microvasculature and hydrodynamics of the uterine supply is still, however, required.

If intravascular coagulation occurs in normal pregnancy, as is suggested, its accentuation in preeclampsia requires explanation. This could be purely a matter of the 'dosage' and 'timing' of intravasated thromboplastin and thus akin to an enhanced Shwartzman phenomenon, in which fibrinolytic activity is depressed by prior fibrin deposition. Alternatively the other factors mentioned (hormonal, immunological and pressor) could also operate in an enhancing role. It is not difficult to imagine a wide range of clinical eventualities.

In summary, the aetiology of intravascular coagulation in pregnancy is complex; no one factor can be regarded as proven but entry of thromboplastin into the maternal circulation seems the most likely.

\section{Possible Effects of Intravascular Coagulation in Pregnancy}

If intravascular coagulation occurs throughout pregnancy and is exaggerated in preeclampsia and eclampsia, its potential effects must be considered. In the gross forms microvascular obstruction is obvious and there is abundant evidence of this. Once again it is the minor degrees that pose problems.
It is difficult to assert that fibrin deposition in renal glomerular capillaries - of a degree that we cannot as yet demonstrate - could itself, unaided, trigger the renin-angiotensin-aldosterone system. On the other hand an episode of intravascular coagulation could be severe enough to cause more significant renal fibrin deposition over a brief period and so initiate the process, subsequent events depending upon the timing and severity of further episodes of coagulation. The endothelial swelling and blebbing that has already been stressed as a possible consequence of fibrin deposition may well play a greater role in capillary obstruction than the fibrin deposition itself. Other factors, such as plasma volume expansion and elevated hormone levels, are likely to contribute to a multi-factorial aetiology of the hypertension, oedema and proteinuria.

\section{Intravascular Coagulation and the Fetus}

So far nothing has been said of the occurrence and effects of intravascular coagulation in the fetus in utero. The existence of a placental 'watershed' is an attractive hypothesis that permits speculation on the possibility of intravascular coagulation in the fetus. It has been shown experimentally after injury to the hamster placenta (Brown and Stalker, 1969) and postulated for the human fetus (Stark et al, 1968). Fibrin microthrombi are frequently noted in stillbirths and neonatal deaths (Wade-Evans, 1961; Boyd, 1965). Haematological studies support the view that intravascular coagulation may occur in the newborn (Hathaway, 1970).

It has been suggested that the respiratory distress syndrome in the newborn might be related to intravascular coagulation and Hall et al (1973) have described deficiency of pulmonary surfactant in neonatal rabbits given intravenous thromboplastin via the umbilical vein at Caesarean section. Other factors, such as prematurity, come into this area, however, and the question is still open.

\section{Conclusion}

In this paper broad issues have been presented. It is today impossible for any one worker to pretend sufficient expertise to analyse every aspect of intravascular coagulation in pregnancy. In particular no account has been taken of the epidemiology of preeclampsia and eclampsia. Whatever the final answer may turn out to be it must be able to explain the many puzzling epidemiological features of this condition.

The author is also aware of the risks both of oversimplification and also of substituting a study of one component of a disease process for the whole 
disease. Yet from a generalizing approach some features of possible value may have emerged.

In particulır attention has been directed to continuing deficiencies in the present methods for histological demonstration of small amounts of intravascular fibrin. The minor grades of intravascular coagulation can be difficult to detect and the need to study a broad spectrum of the phenomenon in natural disease has been emphasized. Much can be gained by considering the speed and degree of operation of causal agents in pathology - the 'tempo of disease'.

Although final proof may still be needed there is much evidence that intravascular coagulation occurs in normal pregnancy and is accentuated in preeclampsia and eclampsia. It is most probably due to the entry of placental thromboplastin into the circulation. This may be initiated quite simply by the vascular nature of the placenta or by minor damage to it, but other hormonal, immunological or pressor factors may modify the reaction.

Future advances in this field are most likely to stem from work directed at fuller understanding of the structure of fibrin, and hence its staining, the reaction of the endothelial cell to fibrin, in particular the formation of plasminogen activator, the vascular hydrodynamics of placentation and the role of the pregnancy specific proteins.

\section{References}

Banti Devi, Jennison, R. F., and Langley, F. A. (1968). Significance of placental pathology in transplacental haemorrhage. J. clin. Path., 21, 322-331.

Barnhart, M. I., Cress, D. C., Noonan, S. M., and Walsh, R. T. (1970). Influence of fibrinolytic split products. on hepatic release and synthesis of fibrinogen. Thrombos. Diathes. haemorrh. (Stuttg.), Suppl. 39, 143-159.

Bonnar, J. (1973). Blood coagulation and fibrinolysis in obstetrics. Clin. Haemat., 2, 213-233.

Bonnar, J., Prentice, C. R. M., McNicol, G. P., and Douglas, A. S. (1970). Haemostatic mechanism in the uterine circulation during placental separation. Brit. med.J., 2, 564-567.

Boyd, J. F. (1965). Disseminated fibrin thrombo-embolism among stillbirths and neonatal deaths. J. Path. Bact., 90, 53-63.

Brosens, I., Robertson, W. B., and Dixon, H. G. (1967). The physiological response of the vessels of the placental bed to normal pregnancy. J. Path. Bact., 93, 569-579.

Brown, L. J., and Stalker, A. L. (1969). The maternal and foetal microcirculation following placental separation or trauma. Microvasc. Res., 1, 403-409.

Brown, L. J., Stalker, A. L., and Hall, J. (1969). Experimental defibrination: histological studies by light and electron microscopy. Microvasc. Res., 1, 295-307.

Copley, A. L., (1957). Fibrinolysis and atherosclerosis. Lancet, 1, 102-103.

Copley, A. L. (1960). Apparent viscosity and wall adherence of blood systems. In Flow Properties of Blood, edited by A. L. Copley and G. Stainsby, pp. 97-121. Pergamon Press, Oxford.
Donald, K. J. (1972). An electron-microscope study of defibrination during carbon clearance in rabbits stimulated by a tubercle bacillary lipid. J. Path., 108, 329-333.

Doolittle, R. F. (1973). Structural aspects of the fibrinogen to fibrin conversion. Advanc. Protein Chem., 27, 1-109.

Gitlin, D., and Craig, J. M. (1957). Variations in the staining characteristics of human fibrin. Amer. J. Path., 33, 267-283.

Goodall, H. B. (1973). Giant nuclear masses in the lungs and blood in malignant malaria. Lancet, 2, 1124-1126.

Govan, A. D. T. (1954). Renal changes in eclampsia. J. Path. Bact., 67, 311-322.

Govan, A. D. T. (1973). The pathology of pre-eclampsia and eclampsia. In Postgraduate Obstetrical and Gynaecological Pathology, edited by H. Fox and F. A. Langley, pp. 375-390. Pergamon Press, Oxford.

Hall, C. E., and Slayter, H. S. (1959). The fibrinogen molecule: its size, shape and mode of polymerization. J. biophys. biochem. Cytol., 5, 11-17.

Hall, J., Macpherson, J. E., and Stalker, A. L. (1973). Neonatal pulmonary changes in experimental D.I.C. in the rabbit. [7th Europ. Confr. Microcirculation, Aberdeen, 1972, Part II.] Bibl. anat. (Basel), 12, 35-40.

Hathaway, W. E. (1970). Coagulation problems in the newborn infant. Pediat. Clin. N. Amer., 17, 929-942.

Huxley, M., Beyts, W., and Stalker, A. L. (1975). Abnormal fibrin formation in pregnancy suggesting D.I.C. [8th Europ. Confr. Microcirculation, Le Touquet,[1974.] Bibl. anat. (Basel), in press.

Lendrum, A. C., Fraser, D. S., Slidders, W., and Henderson, R. (1962). Studies on the character and staining of fibrin. J. clin. Path., 15, 401-413.

McKay, D. G. (1965). Disseminated Intravascular Coagulation - An Intermediary Mechanism of Disease. Hoeber, New York.

Marder, V. J. (1971). Identification and purification of fibrinogen degradation products produced by plasmin: considerations on the structure of fibrin. Scand. J. Haemat., Suppl. 13, 21-36.

Moe, N., and Abildgaard, U. (1969). Histological staining properties of in vitro formed fibrin clots and precipitated fibrinogen. Acta path. microbiol. scand., 76, 61-73.

Mole, R. H. (1948). Fibronolysin and the fluidity of the blood post mortem. J. Path. Bact., 60, 413-427.

Ogston, D. (1973). Haemostasis and other homeostatic mechanisms. Clin. Haemat., 2, 53-63.

Petrucco, O. M., Thomson, N. M., Lawrence, J. R., and Weldon, M. W. (1974). Immunofluorescent studies in renal biopsies in pre-eclampsia. Brit. med. J., 1, 473-476.

Pirani, C. L., Pollak, V. E., Lannigan, R., and Folli, G. (1963). The renal glomerular lesions of pre-eclampsia: electron microscopic studies. Amer. J. Obstet. Gynec., 87, 1047-1070.

Robertson, W. B., Brosens, I., and Dixon, H. G. (1967). The pathological response of the vessels of the placental bed to hypertensive pregnancy. J. Path. Bact., 93, 581-592.

Ryan, T. J. (1972). Mechanisms of vascular disease in the skin. Fibrin and fibrinolysis. In Methods in Microcirculation Studies, edited by T. J. Ryan, B. Jolles, and G. Holti, pp. 85-91. Lewis, London.

Schneider, C. L. (1952). Rupture of the basal (decidual) plate in abruptio placentae: a pathway of autoextraction from the decidua into the maternal circulation. Amer.J. Obstet. Gynec., 63, 1078-1090.

Simpson, J. G., Hall, J., and Stalker, A. L. (1971). Histological changes in defibrination: experimental and clinical. J. Path., 103, (Abstr). p. ix.

Simpson, J. G., Mackie, M. J., Hamlet, M. E., and Stalker, A. L. (1973). Intravascular coagulation and plasma fibrinogen in pregnancy. [7th Europ. Confr. Microcirculation, Aberdeen, 1972, Part II.] Bibl. anat. (Basel), 12, 6-12. 
Stalker, A. L. (1973). The entry of placental thromboplastin into the maternal and fetal circulations. [7th Europ. Confr. Microcirculation, Aberdeen, 1972, Part II.] Bibl. anat. (Basel), 12, 55-57.

Stark, C. R., Abramson, D., and Erkan, V. (1968). Intravascular coagulation and hyaline-membrane disease of the newborn. Lancet, 1, 1180-1181.

Thomson, D., Paterson, W. G., Smart, G. E., Macdonald, M. K., and Robson, J. S. (1972). The renal lesions of toxaemia and abruptio placentae studied by light and electron microscopy. J. Obstet. Gynaec. Brit. Cwlth, 79, 311-320.
Todd, A. S. (1959). The histological localisation of fibrinolysin activator. J. Path. Bact., 78, 281-283.

Todd, A. S. (1971). Endothelial fibrinolysis and blood-flow. (Letter.) Lancet, 1, 1179.

Vassalli, P., Morris, R. H., and McCluskey, R. T. (1963) The pathogenic role of fibrin deposition in the glomerular lesions of toxemia of pregnancy. J. exp. Med., 118, 467-477.

Wade-Evans, T. (1961). Thrombi in the hepatic sinusoids of the newborn and their relation to pulmonary hyaline membrane formation. Arch. Dis. Childh., 36, 286-292.

Woodfield, D. G., Cole, S. K., Allan, A. G. E., and Cash, J. D. (1968). Serum fibrin degradation products throughout normal pregnancy. Brit. med. J., 4, 665-668. 